\title{
Aplicación de un LMS como herramienta de b-learning en estudios de posgrado
}

\author{
Eufrasio Pérez Navío*, Laura Herrera Corona**, Guadalupe Aurora Maldonado Verea**, \\ Norma Elena Mendoza Zaragoza**
}

\section{Resumen}

Incorporar tecnología de b-learning en una universidad implica no solamente el conocimiento de la tecnología por sí misma, sino una serie de habilidades que necesitan ser aprendidas, desarrolladas y apropiadas para los estudiantes que participan en el proceso. Supone el fortalecimiento de la expresión escrita, la experiencia en la investigación, el trabajo colaborativo, así como el perfeccionamiento de las aptitudes en informática.

Actualmente, un estudiante b-learning necesita ser multidisciplinario. Esta investigación describe la integración de Moodle en la Universidad Cristóbal Colón a un ambiente oficial de b-learning como una herramienta de apoyo en clases presenciales de nivel Maestría. Fue empleada una metodología híbrida para indagar habilidades y competencias tecnológicas desarrolladas por 98 estudiantes al utilizar y evaluar Moodle, así como las ventajas y las desventajas que identificaron, tanto desde el punto de vista tecnológico como pedagógico. La muestra fue no probabilística de sujetos tipo, abarcando las áreas de Educación, Tecnologías de Información, Redes y Telecomunicaciones. Los estudiantes fortalecieron varias habilidades cognitivas y metacognitivas, mismas que fueron apoyadas por la tecnología. Algunas de dichas actividades son: análisis, observación, reflexión, abstracción de ideas, solución de problemas, autoestudio, comprensión de textos y expresión escrita.

\footnotetext{
Abstract

Incorporating b-learning technology in a University involves not only the knowledge of the technology itself, but a series of skills that need to be learned, developed and appropriate for students who participate in the process. This would bring strength in written expression, experience in research, collaborative work and the development of skills in computer science.
}

Currently, a b-learning student needs to be multidisciplinary. This research describes the integration of Moodle at the University Cristóbal Colón to an environment as official b-learning and support Masterslevel classrooms. A hybrid methodology was employed to investigate technological skills and competencies developed by 98 students to use and evaluate Moodle, as well identified the advantages and disadvantages, both from the technological and pedagogical points of view. It was a non-probabilistic simple type of subjects, covering the areas of Education. Information Technology, Networks and Telecommunications. Students strengthen various cognitive and "metacognitive" skills, which were supported by technology. Some of these activities are: analysis, observation, reflection, abstraction of ideas, problem solving, self-study, reading comprehension and writing.

\section{Introducción}

La Universidad Cristóbal Colón (UCC) persigue, mediante su modelo educativo, hacer frente a las prerrogativas que han impactado de manera significativa al área educativa, por un lado; y a las exigencias y prioridades que solicita el mercado laboral, por el otro. Esto requiere necesariamente la integración de tecnología en los procesos didácticos de todos los niveles educativos.

A partir de estas necesidades es que la UCC se plantea la puesta en marcha de un programa de aplicación de tecnología educativa que implicó varias fases. En primer lugar, un diagnóstico de habilidades tecnológicas que involucró a toda la población (maestros y estudiantes) de la Universidad.

En segundo lugar una evaluación entre plataformas para seleccionar la más apropiada con la definición de los ejes transversales que permean al modelo educativo de la UCC. La elección de dicha plataforma se realizó

* Eufrasio Pérez Navío. Universidad de Jaén. eufrasionavio@hotmail.com

** Laura Herrera Corona, Guadalupe Aurora Norma Maldonado Verea, Elena Mendoza Zaragoza.Universidad Cristóbal Colón. Veracruz, México. 
mediante un proceso de benchmarking entre cuatro opciones de software libre y propietario: Flé, Mimer, Blackboard y Moodle. Se tomaron en cuenta los siguientes aspectos desglosados en 175 indicadores: Herramientas de Comunicación, Herramientas de Productividad, Herramientas que involucran al estudiante, Herramientas de Administración, Herramientas de Difusión del Curso, Herramientas de Diseño del Currículum, Especificaciones Técnicas de Hardwarey Software y Especificaciones de Precio y Licencia.

En tercer lugar, una vez que Moodle fue seleccionada como el recurso oficial de blearning ${ }^{1}$ en la UCC, se elaboraron una serie de manuales para establecer los criterios de diseño para los cursos que iban a estar disponibles en dicha plataforma, tanto desde el punto de vista de los contenidos (orden de las unidades, tipos de actividades y manera de utilizarlas, estrategias didácticas posibles) como desde la concepción de los elementos visuales y gráficos a emplear (tipos de letra, colores, uso de las imágenes). En paralelo se diseñaron cursos de capacitación docente en Tecnología educativa: Internet y bibliotecas virtuales; estrategias didácticas constructivistas; software de ofimática y educativo; y uso y aplicación de Moodle (al inicio como alumno, posteriormente, como docente).

La implementación de la herramienta seleccionada de e-learning implica el conocimiento de las competencias tecnológicas que tienen los docentes y los estudiantes, previos al contacto con este tipo de ambientes. La implementación de los cursos de Maestría apoyados por b-learning se basa en contenidos y actividades específicas, que corren a cargo del docente. Por lo que su planeación, así como las estrategias didácticas (concebidas con una proyección a la vida profesional del estudiante) pueden resultar muy atinadas y en ocasiones, muy desafortunadas. Todo depende de la capacitación que posee el profesor, tanto en lo didáctico como en el empleo de la tecnología (Tecnología educativa). Si el facilitador no ha sido capacitado de una forma integral, entonces las actividades en ocasiones provienen de su intuición o de experiencias previas en el campo de lo presencial. Es por ello que en la práctica de b-learning, el uso, las percepciones, los resultados y el impacto en los alumnos pueden resultar muy distintos.

Esta investigación se basa en la aplicación de Moodle en cursos de Maestría por parte de profesores capacitados en Tecnología Educativa, y la percepción que se generó en los alumnos a partir de dicha experiencia.

\section{Marco Teórico. E-learning y la educación en línea}

A raíz de la adopción generalizada del Internet como medio de transmisión de información, la forma como se conciben las aplicaciones educativas experimentan una profunda transformación al permitir ese intercambio pluridireccional de mensajes, entre un amplio número de usuarios, enriqueciendo así el proceso de la comunicación en el ámbito de la educación:

La utilización de las diferentes redes de telecomunicación como soporte para sistemas de enseñanza a distancia ha permitido establecer un canal de retorno desde los alumnos a los profesores, incorporando un valor añadido importantísimo. La combinación de la telecomunicación y la enseñanza ha permitido acuñar el término de enseñanza asistida por computadora o e-learning. (Anido-Rifón, 2001).

"e-learning es el uso de tecnología de redes para el diseño, entrega, selección, administración y extensión del aprendizaje" (Masie, 2007). E-learning traslada las experiencias de aprendizaje fuera de la tradicional aula de clases. En forma general, el término e-learning se refiere ampliamente al aprendizaje basado en tecnología; actualmente parece enfocarse en métodos basados en Web, pero frecuentemente es usado en su más amplio contexto. De estas definiciones, se pueden deducir tres grandes componentes en los procesos de e-learning:

1) Las tecnologías de soporte a los procesos de aprendizaje (redes, hardware, software y herramientas en forma general),

2) Los contenidos o elementos contenedores de información y,

3) Las personas que interactúan en el proceso de aprendizaje y de soporte al mismo.

La característica principal de los nuevos entornos de aprendizaje virtual, reside en la utilización de la Web como única plataforma de distribución. Así, desaparecen los problemas de tiempo, espacio y horarios. Los estudiantes pueden repasar y acceder a contenidos educativos desde cualquier lugar y a cualquier hora, ya que los sistemas de enseñanza asistida por computadora están disponibles las 24 horas de todos los días del año.

Por otro lado, las plataformas en línea proporcionan un canal abierto de comunicación entre estudiantes y 
profesores, permitiendo la participación de personas que pueden encontrarse en cualquier lugar del mundo, lo que enriquece el proceso de intercambio cultural. Es posible ofrecer entornos de telepresencia, donde todos los participantes en el proceso formativo compartan un

Dado que el ámbito de actuación de sistemas educativos en línea es universal, los estudiantes pueden elegir entre una gran diversidad de materias, cursos y especialidades. Así, estudiantes y maestros pueden estar distribuidos geográficamente sin que esto afecte el acceso a los contenidos de aprendizaje, incluso pueden intercambiar puntos de vista, retroalimentar sobre calificaciones, hacer trabajo colaborativo, etcétera. Un aspecto elemental a considerar es que esta forma de aprendizaje requiere de un esfuerzo significativo por parte de los actores involucrados. El docente, principalmente, debe estar atento de no perder el contacto con los alumnos de su curso, de darle pronta respuesta a las consultas que éstos pudieran hacerle, de actualizar las actividades y contenidos en la plataforma virtual de aprendizaje cuando considere oportuno o necesario, según perciba el desarrollo grupal en ese curso en particular. Por otro lado la tarea del estudiante de manera que pueda funcionar como autodidacta y cumplir con las metas establecidas desde el principio hasta el final del proceso. aula virtual. será imprescindible, ya que deberá administrar su tiempo

Resulta esencial para nuestros fines comentar algunas de las problemáticas que aparecen a raíz de la proliferación de los programas de estudio online: la heterogeneidad de las plataformas disponibles; la interacción entre personas y sistemas; el aumento en la información disponible, así como su desviación y pérdida motivada por la dispersión geográfica de los usuarios de los cursos.

\section{Los procesos que involucra e-learning}

De acuerdo con Comezaña y García (2005), el diseño de contenidos para e-learning implica estructurar conocimiento desde dos tipos de procesos: los procesos técnicos y los procesos pedagógicos. Lo anterior implica que es necesario trabajar desde el diseño de sistemas, programación, comunicación, privacidad, etcétera, como desde la concepción de contenidos de aprendizaje que impliquen dichas herramientas tecnológicas pero con objetivos, actividades, formas de evaluación y retroalimentación bien definidas y diseñadas. Para esquematizar lo anteriormente dicho, se presenta la figura 1.

El esquema anteriormente presentado, no deja de ser interesante, sin embargo se considera que resultaría importante reconocer y agregar, del lado de los procesos pedagógicos, los diferentes estilos de aprendizaje, así como los diversos canales de comunicación que tiene el educando, pues éstos determinan la manera en cómo ve, percibe,

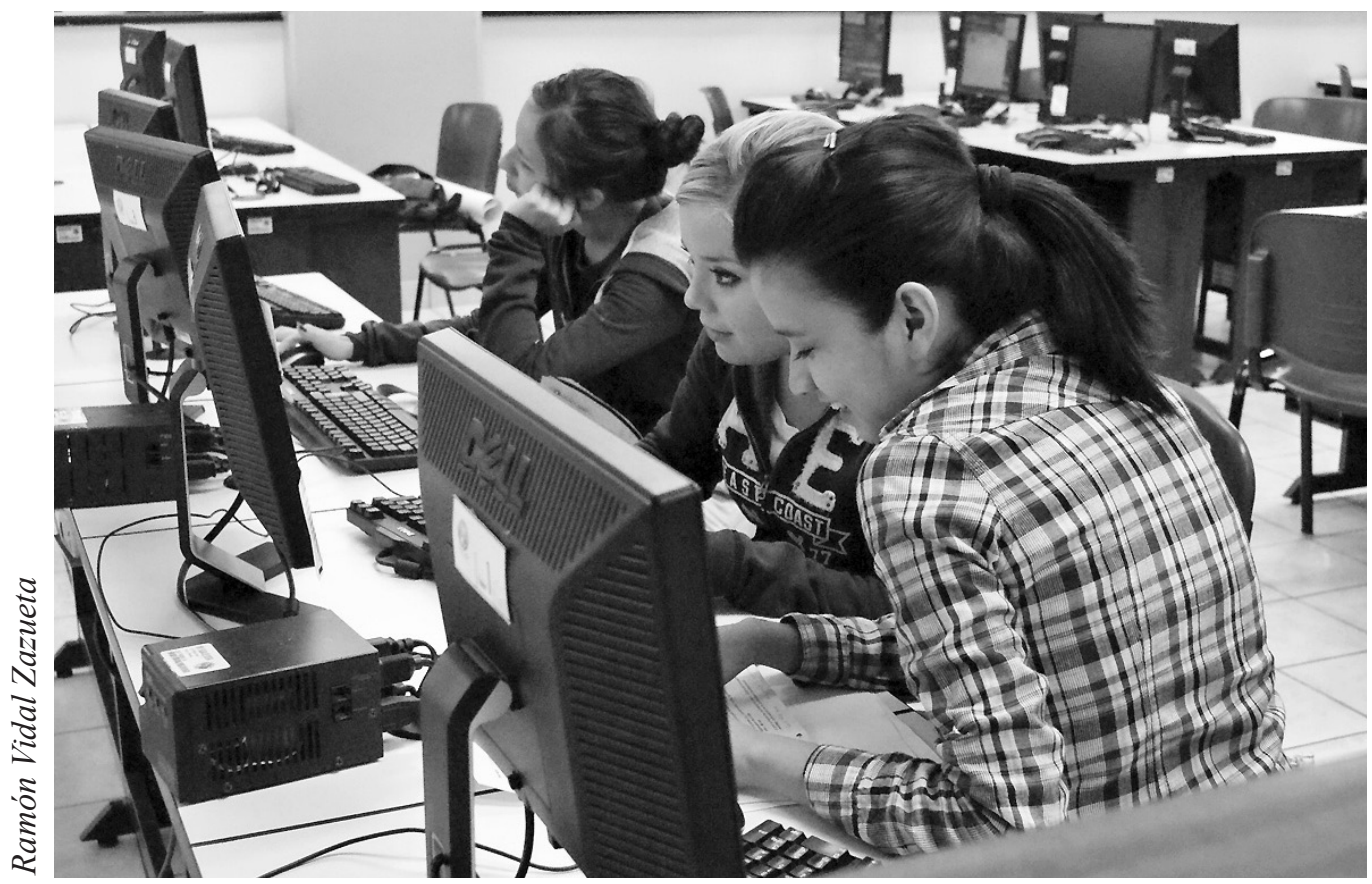
concibe e interactúa con el mundo; por lo tanto, es conveniente que al identificar dichas particularidades, se le proporcione un modelo que pueda facilitar su proceso de aprendizaje de manera proactiva, incluyente e integral. A partir de ese conocimiento cercano del alumno, es posible la personalización del proceso educativo, que se verá claramente reflejada en la selección de las técnicas didácticas específicas a utilizar 


\section{旗 Sonta}

Figura 1. El proceso de e-learning

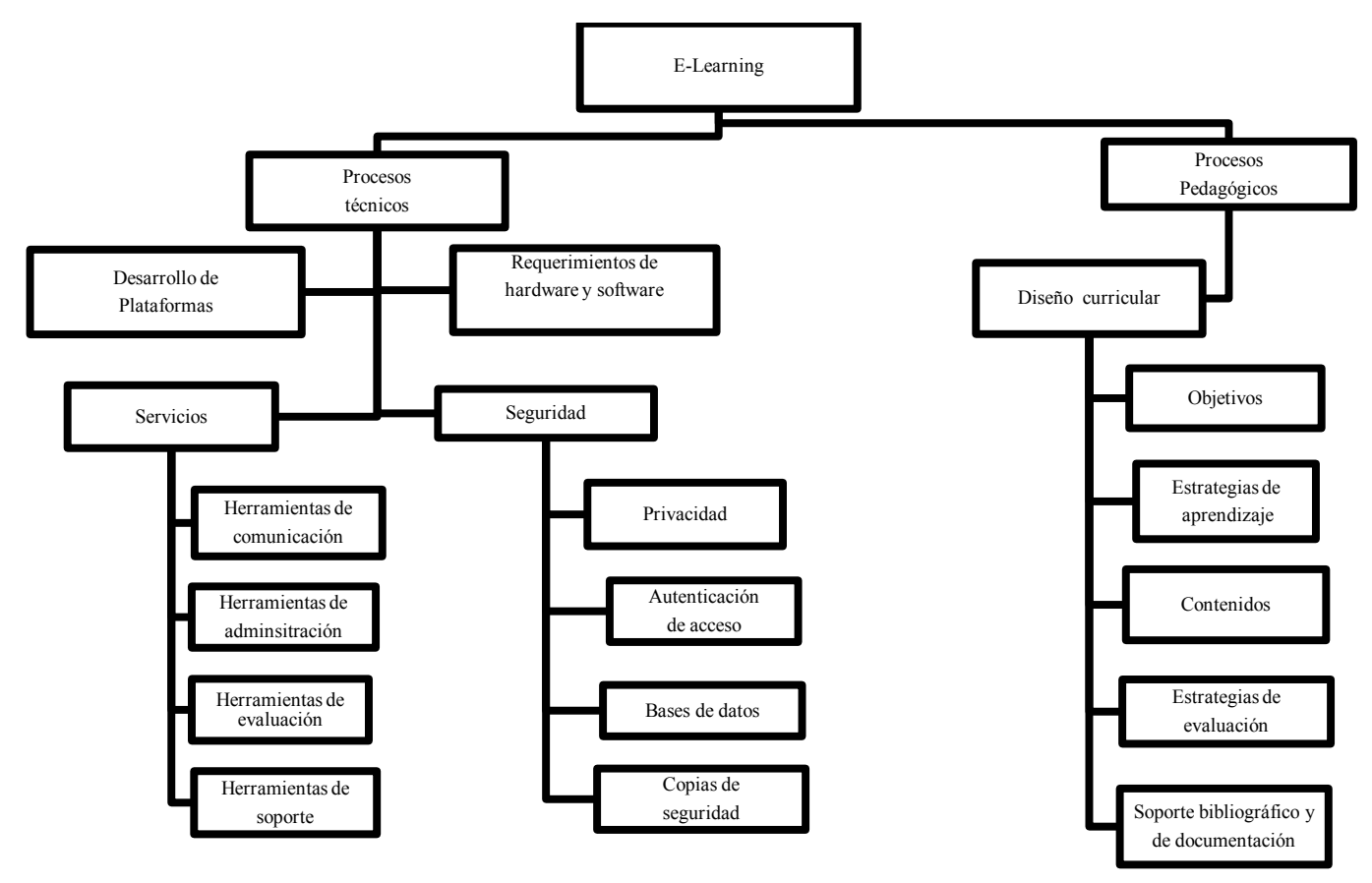

Fuente: Comezaña y García $(2005,1)$

durante un proceso de e-learning, tales como el aprendizaje colaborativo, el aprendizaje basado en problemas, el aprendizaje orientado a proyectos, el aprendizaje basado en la construcción del conocimiento, entre otras, así como las formas de auto y coevaluación que dichas estrategias llevan implícitas.

\section{Objetivos}

- Identificar las herramientas de e-learning que los estudiantes de Maestría de la UCC han utilizado.

- Identificar las herramientas de e-learning que los usuarios de la UCC conocen y dominan.

- Indagar las ventajas y las desventajas, tanto desde el punto de vista tecnológico como pedagógico, que los usuarios encuentran en Moodle.

- Explorar las habilidades y competencias que los alumnos de Maestría de la UCC han desarrollado a partir de la utilización de la herramienta e-learning en cuestión.

- Identificar inquietudes y necesidades de capacitación y adiestramiento tecnológico que los estudiantes de la UCC manifiestan.

\section{Método y proceso de investigación}

La presente etapa de investigación se abordó desde la perspectiva de la teoría fundamentada, según la cual, la construcción de conceptos y el desarrollo de conocimientos se da a partir de la propia experiencia de los sujetos investigados. La técnica de acopio de información utilizada fue el cuestionario con preguntas de opción múltiple y abiertas, en el cual se indagaron aspectos como: ventajas y desventajas encontradas a partir del trabajo con Moodle, desde el punto de vista tecnológico y pedagógico; competencias desarrolladas; recomendaciones de utilización que se hicieron a otros estudiantes y a los mismos profesores implementadores de cursos desarrollados con esta herramienta. Para analizar los datos se combinó la metodología cuantitativa (para distribución de frecuencias) y cualitativa (en la construcción de categorías de análisis y la interpretación de la información). El cuestionario fue aplicado a una muestra no probabilística de sujetos tipo de 98 estudiantes, que cursaron la Maestría en la UCC, durante el periodo de 2006-2007. Con el fin de obtener las diversas percepciones, a partir de la práctica educativa apoyada por Moodle, se seleccionaron tres cursos: de la Maestría en Educación, la asignatura de Tecnología y enseñanza; de la Maestría en Tecnologías 
de Información la materia del Taller de investigación, módulo I: Redacción de textos científicos; y de la Maestría en Redes y Telecomunicaciones, la asignatura de Introducción a la investigación. Los grupos se encontraban geográficamente dispersos, algunos en el campus Torrente Viver de la Universidad, ubicado en Veracruz, Veracruz (México); y otros, en el Instituto Tecnológico Superior de San Andrés Tuxtla, Veracruz (ITSSAT).

\section{Resultados}

Para los fines del proyecto de Tecnología Educativa en la Universidad Cristóbal Colón, ha resultado de gran valía e interés analizar los resultados de la presente investigación elaborada a nivel Maestría, ya que, además de probar y utilizar Moodle como herramienta de e-learning para ser implementada en un futuro cercano en las modalidades de educación en línea, se tuvo la oportunidad de obtener opiniones, puntos de vista y perspectivas desde diferentes niveles, áreas geográficas y campos de conocimiento.

Podemos resaltar, como primer aspecto relevante, que de las herramientas que los 98 alumnos entrevistados han utilizado, Moodle es la que resalta como más práctica, rápida, versátil y fácil de aprender y utilizar. Algunas de las herramientas con las cuales los estudiantes compararon Moodle fueron: Cisco Networking Academy Program, Blackboard, Mimer Desk, Lotus Learning Management System y Claroline.

Es importante mencionar que el conocimiento de otras plataformas de e-learning fue más ampliamente encontrado en la Maestría en Tecnologías de Información que se imparte en el Campus Torrente Viver de la UCC, donde más del 53\% ya había utilizado alguna otra herramienta de e-learning, a diferencia de la misma Maestría impartida en la plaza de San Andrés, Tuxtla, donde el $80 \%$ no conocía herramientas de esta naturaleza. En la Maestría en Educación, el 88\% de la población se encontraba en desconocimiento de alguna de ellas.

\section{Desde lo tecnológico}

En cuanto a las ventajas que el nivel tecnológico presenta Moodle, en la Maestría en Educación se resaltaron aspectos como la sencillez de su aprendizaje y uso, así como la versatilidad en el manejo de tiempos y espacios de aprendizaje, la facilidad para acceder a contenidos, tareas, exámenes desde cualquier tiempo y lugar. Así mismo, se mencionó que permite la integración y recuperación rápida de la información, facilita la planeación y organización de los cursos, permite la administración eficiente de la información, optimiza tiempos, facilita la comunicación entre maestros y alumnos y permite el desarrollo de la creatividad por parte del que planea los cursos.

En la Maestría en Tecnologías de Información impartida en San Andrés Tuxtla, los alumnos resaltaron como ventaja tecnológica la facilidad que Moodle otorga a la comunicación entre maestros y alumnos, situación que para ellos es de suma importancia al encontrarse geográficamente distantes de su profesor, y donde, gracias a la herramienta pudieron acceder a contenidos, actividades calendarizadas, foros de discusión y más. Se refirieron a las ventajas que se pueden tener sobre el correo electrónico, la incompatibilidad entre algunos servidores, el tráfico de información, etcétera. Se habló también de la integración y recuperación rápida de la información, la fácil navegación y el sencillo uso de la herramienta que les permitió contar siempre con ayuda en línea y fuera de ésta. Se mencionó también la administración eficiente de la información, la comodidad, la vanguardia y el hecho de contar con una herramienta de libre distribución.

En la Maestría de Tecnologías de la Información, pero impartida en la Ciudad de Veracruz, encontramos que en el nivel tecnológico se destaca nuevamente la facilidad de la comunicación maestro-alumno, a pesar de que ellos se encontraban físicamente ubicados en el mismo lugar que la docente. También citaron la importancia de contar con una plataforma de open source y robustamente respaldada por el lenguaje php que utiliza. Resaltaron también la facilidad de acceder a los contenidos desde cualquier lugar y a cualquier hora, lo que proporciona comodidad. Se mencionó también que facilita el aprendizaje virtual y que es una herramienta de apoyo al conocimiento. Se resaltó la administración eficiente de la información, misma que facilita el trabajo docente, quien puede tener un mayor control de los procesos.

\section{Desde lo pedagógico}

En el sentido pedagógico resulta de gran relevancia revisar las diferencias encontradas entre los puntos de vista de un área como la educativa y una como la tecnológica, mismas que, en un proceso ideal de 


\section{就 Sorvida}

e-learning debieran complementarse y fusionarse en un trabajo interdisciplinario para lograr buenos resultados.

En la Maestría en Educación los estudiantes consideran que Moodle convierte el proceso de aprendizaje en algo más interactivo, interesante y atractivo; además que fomenta la interacción y que resulta motivador porque capta la atención de los estudiantes de muy diversas maneras. Permite la integración de una gran variedad de recursos, actividades y documentos, así como la interacción con otras paqueterías. Otro aspecto muy interesante que se mencionó fue que modifica el rol del profesor y del estudiante, convirtiéndose el primero más en un facilitador y el segundo en un ser responsable de su propio aprendizaje. Entre los aspectos pedagógicos que se tocaron están que fomenta el pensamiento creativo y reflexivo, así como el aprendizaje autónomo y la construcción del conocimiento.

Por otro lado, en la Maestría en Tecnologías de Información, desde lo pedagógico nuevamente se resaltó la rapidez y accesibilidad de la comunicación entre los integrantes del proceso educativo. Se puntualizó también sobre el fomento del autoaprendizaje y el estudio independiente, así como la versatilidad en el manejo de los tiempos y los espacios para programar sus actividades, además del control y seguimiento de los alumnos que esta herramienta ofrece.
En la Maestría en Redes y Telecomunicaciones, Campus Torrente Viver, se resaltaron aspectos parecidos que en la plaza de San Andrés Tuxtla, pues se habló de la accesibilidad de la comunicación, también se discutió en una forma más profunda sobre el proceso de aprendizaje, la construcción del conocimiento que se da en cada estudiante, la aparición de un proceso de enseñanza más interactivo basado en habilidades y conocimientos propios. También en este orden, citaron al Moodle como una herramienta innovadora que facilita las cosas, porque gestiona recursos educativos proporcionados por el profesor; organiza el acceso a esos recursos por el alumnado; se puede mostrar información de una forma entendible para el usuario y facilita el aprendizaje a distancia.

Por último, pero no menos importante, disertaron que la plataforma virtual fomenta el aprendizaje autónomo, que convierte la clase en un proceso más dinámico, interactivo y variado que da al método de aprendizaje un valor agregado.

\section{Algunas desventajas encontradas}

Como críticos de los recursos que utilizan, se les cuestionó a los alumnos qué desventajas encontraban en la plataforma utilizada. Cada área desde su propia perspectiva e inquietud respondió:

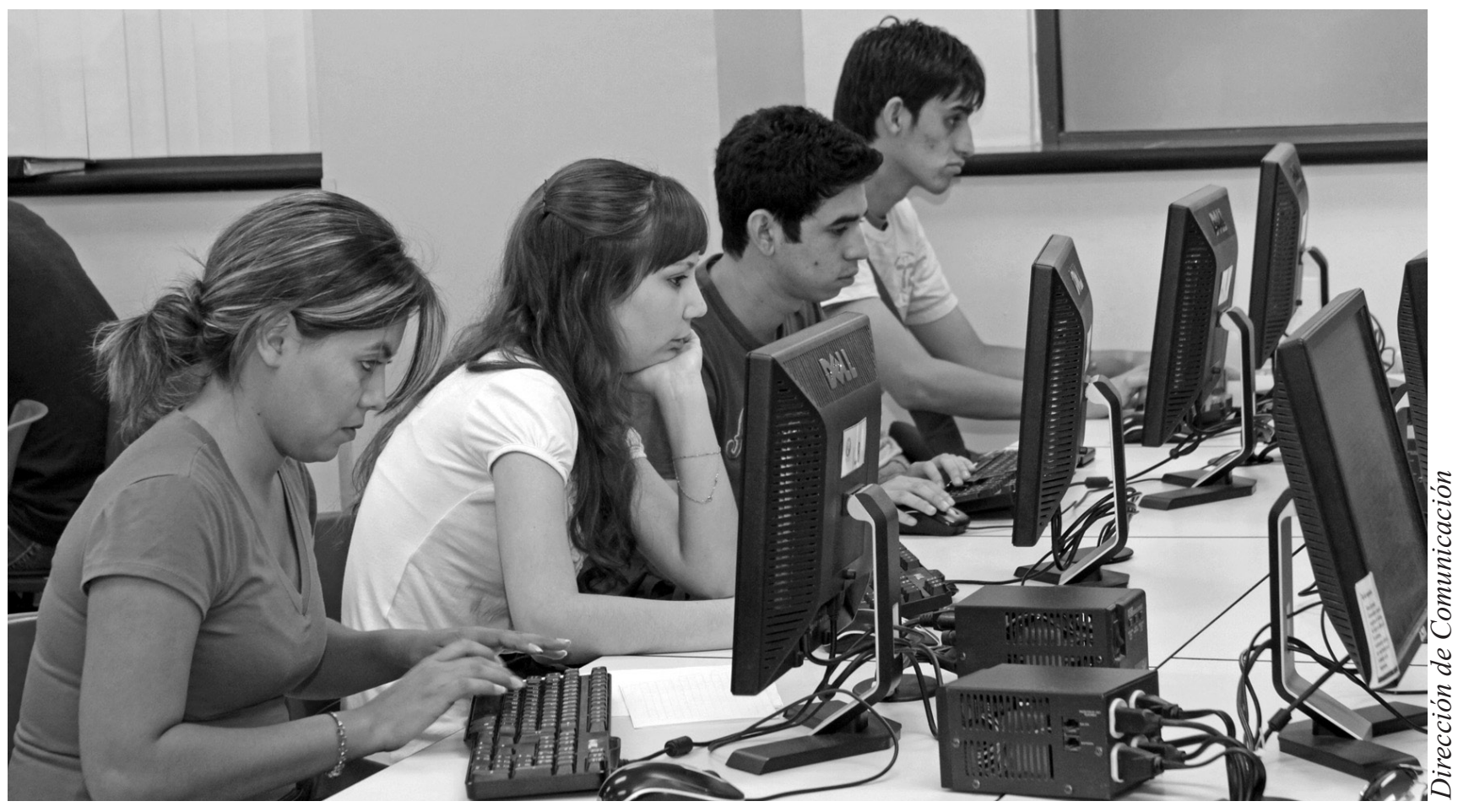


En la Maestría en Educación se localizaron como desventajas la forma en que el proceso de enseñanza puede volverse frío y difícil de monitorear. Les preocupó la falta de contacto profesor-alumno y la incapacidad para dar seguimiento cercano a los participantes de un curso. Se comentaron también dos aspectos más bien relacionados con el formato: el tamaño de los archivos que pueden subirse, que no debe ser mayor a $2 \mathrm{Mb}$ (según las especificaciones dadas por el administrador de la red), así como el diseño visual de la plataforma, que fue calificado como "pobre" y falto de colores más atractivos. Algunos expresaron que el acceso a estos recursos se limita a cierta cantidad de personas que usan y dominan la tecnología computacional; de hecho, una de ellas expresó que no le gustó y no se identificó con ella. Otro comentó que la plataforma limita el proceso de reflexión al ser tan cerrado en sus opciones para manejar cuestionarios.

En San Andrés Tuxtla (Maestría en Tecnologías de Información) en primer lugar, se asume que, por la naturaleza del perfil de los alumnos, se refirieron a la complejidad de la implementación en caso de adoptar esta herramienta, y de las fallas técnicas por parte del servidor de Moodle o de la conexión, que pueden entorpecer el proceso. Entre los comentarios también encontramos que el aspecto humano (tutor o facilitador) cobra relevancia, pues en caso de que éste no esté al pendiente de lo que sucede en la plataforma, con respecto a los comentarios y dudas de los alumnos, el proceso e-a podría no resultar tan efectivo.

Por otro lado, los alumnos de la Maestría en Redes y Telecomunicaciones, cursada en el Campus Torrente Viver, resaltaron como desventaja el uso inapropiado y la falta de cultura por parte de docentes y alumnos para trabajar en esta modalidad de enseñanza. Lo anterior puede estar relacionado con la resistencia que este grupo en concreto presentó al inicio del curso, pues ningún participante se había visto involucrado en un proceso bajo esta modalidad, ni en las demandas que dicho proceso conlleva.

Otra desventaja mencionada por los tres grupos de Maestría fue que dependemos de Internet para poder utilizar la herramienta, cosa que no sucede con otras plataformas como LLMS que permiten el manejo Offline, para poder actualizar contenidos con posterioridad.

\section{Competencias desarrolladas a partir del trabajo con Moodle}

Las personas que cursan la Maestría en Educación resaltaron entre las competencias desarrolladas a raíz de la utilización de Moodle, en primer lugar el haber adquirido más conocimientos de computación y específicamente, en el campo del e-learning, así como una mejor comprensión de las posibilidades que la tecnología ofrece en el campo de la enseñanza. Así mismo se resaltó la destreza en el manejo de recursos tecnológicos y el dominio de otras paqueterías que antes no dominaban. Los estudiantes y la mayoría de los profesores encontraron haber desarrollado varias habilidades cognitivas y metacognitivas que fueron apoyadas por la tecnología, tales como: análisis, observación, reflexión, abstracción de ideas, solución de problemas, comprensión de textos y expresión escrita. Se mencionó también, con énfasis, el desarrollo de la creatividad como materia prima para la producción de materiales didácticos, así como esa posibilidad del programa para permitir la comunicación y el trabajo colaborativo en equipo con el fin de lograr los objetivos de aprendizaje establecidos. También se resaltó la posibilidad de la plataforma para permitir la rapidez, discriminación y búsqueda atinada de información según las necesidades del usuario.

En la Maestría en Tecnologías de Información, impartida en San Andrés Tuxtla, los estudiantes resaltaron, en primer lugar, haber adquirido las competencias necesarias de análisis, autodidactismo, autoestudio, abstracción de ideas, comprensión de textos, lectura, iniciativa de cultura e investigación, todas ellas necesarias para el logro del objetivo del curso, competencias que fueron fomentadas y apoyadas por la estructura y los requerimientos del mismo. En segundo lugar se encontró que los estudiantes dicen haber adquirido competencias en búsqueda y discriminación de información, comentarios que se deben en gran parte a la naturaleza del curso que se impartió en este grupo. En tercer lugar se mencionan los conocimientos adquiridos en tecnología de e-learning y la conciencia de las posibilidades que la tecnología ofrece para el propio desarrollo y el de los cursos que puedan tomar o impartir.

En el grupo de Maestría en Redes y Telecomunicaciones, localizado en Veracruz, encontramos respuestas coincidentes; además de resaltar la posibilidad de flexibilizar el acceso en diversos tiempos dentro del proceso de e-a, y de garantizar ambientes de aprendizaje 


\section{落 Sonta}

colaborativo mediante el uso de herramientas de comunicación.

\section{Recomendamos a otros profesores utilizar Moodle}

Cabe resaltar que el $100 \%$ de los estudiantes de la Maestría en Educación, el 100\% de los de la Maestría en TI (San Andrés) y el 92.3\% de los alumnos de la Maestría en Veracruz, declararon que sí recomendarían a otros profesores utilizar Moodle en la impartición de sus clases, ya sea en modalidad presencial, semipresencial o a distancia.

Al terminar el cuestionario se preguntó a los alumnos sobre su experiencia al haber tomado una clase apoyada por Moodle como herramienta de e-learning, a lo cual respondieron con adjetivos como: gratificante, enriquecedora, grata, útil, práctica, dinámica, fácil, rápida, cómoda, entre otros.

\section{Beneficios}

Al hablar de los beneficios que se obtendrán, a mediano y largo plazo, a partir de la integración de tecnología de e-learning podemos mencionar: Para la Universidad y la Sociedad: Prestigio y reconocimiento al proveer de egresados con las experiencias tecnológicas y computacionales apropiadas, como lo harían "en la vida real", que sepan discriminar información, que sepan trabajar de manera colaborativa (virtual o presencial) y que se puedan expresar de una forma adecuada, de manera oral y escrita.

Para el alumno: El desarrollo del aprendizaje significativo, experimentar una formación que lo prepare para el mundo real, autoaprendizaje, productividad, desarrollo del pensamiento crítico y creativo, empleo de tecnología.

Para el maestro: lograr un mejor trabajo, librándolo de labores secretariales, de trabajos tediosos y rutinarios; hacer más efectivo el tiempo invertido, incrementando la productividad, tener las bases empíricas para evaluar su propia efectividad docente, contar con un ambiente en el que pueda organizar y preparar sus materiales y tareas para la clase, y lo más importante, descubrir e integrar materiales y métodos optimizados, reduciendo idealmente, las barreras entre la docencia y la investigación.

\section{Conclusiones}

Los sistemas de e-learning tienen una dualidad pedagógica y tecnológica. Pedagógica en cuanto a que estos sistemas no deben ser meros contenedores de información digital, sino que dicha información debe ser transmitida de acuerdo con unos modelos y patrones pedagógicamente definidos para afrontar los retos de estos nuevos contextos. Tecnológica en cuanto que todo el proceso de enseñanza-aprendizaje, se sustenta en aplicaciones software, principalmente desarrolladas en ambientes $W e b$.

El reto actual para la UCC, y los miembros de su plantilla docente, es crear cambios significativos en sus estrategias de enseñanza, con la finalidad de llevar a cabo un apropiado uso de tecnología como una herramienta de apoyo, pero no como un sustituto de los actores principales en el proceso educativo (estudiantes, maestros, asesores). Lo que se pretende es un proceso de comunicación constante, escenarios de intercambio y colaboración, procurando construir un aprendizaje significativo centrado en el alumno. Conforme los estudiantes progresen gradualmente necesitarán menos instrucciones de tipo didáctico para hacer más atractivo el aprendizaje activo, así como una atmósfera donde los estudiantes tomen la responsabilidad de su propio aprendizaje y los instructores tomen el rol de asesores en casos de estudio, simulaciones, tareas, y proyectos colaborativos donde se aproxime a los estudiantes a experimentar una formación profesional que lo prepare para el mundo real.

\section{Notas}

1 Formación combinada, del inglés blended learning. Consiste en un proceso docente semipresencial; esto significa que un curso en este formato incluirá tanto clases presenciales como actividades de e-learning.

2 Considerada como una de los LMS (Learning Management Systems, Sistemas Administrativos del Aprendizaje) más eficientes en el logro de un trabajo con pedagogía constructivista y de carácter colaborativo.

\section{Bibliografía}

Adelsberger, H. and Bick, Markus and Pawlowski, Jan M., (2000). Learning environments for simulation education: design principles for teaching simulation with explorative learning environments. WSC .00: Proceedings of the 32nd 


\section{Sonte}

conference on Winter simulation. San Diego, CA, USA: Society for Computer Simulation International. pp. 1684-1691

Barverá, E. et al. (2001). Enseñar y aprender a distancia: ¿es posible? Disponible en: http://www.uoc.edu/ web/esp/art/uoc/0105018/ensapren.html Fecha de última consulta 2 de Diciembre de 2004

Benbunan-Fich, R. (2002). Improving education and training with IT. Commun. ACM. Último acceso abril 18, 2005. http://doi.acm. org/10.1145/508448.508454.

Berghel, H. \& Sallach, D. (2004). A paradigm shift in computing and IT education. Commun. ACM. Último acceso abril 18, 2005.

http://portal.acm.org/citation.cfm?id=990685\& coll $=\mathrm{ACM} \& \mathrm{dl}=\mathrm{ACM} \& \mathrm{CFID}=6092754 \& \mathrm{CFT}$ OKEN $=63103570$

Deden, A. (1998). Computers and systemic change in higher education. Commun. ACM. Último acceso abril 18, 2005.

http://portal.acm.org/citation.cfm?id=268116\& coll $=\mathrm{ACM} \& \mathrm{dl}=\mathrm{ACM} \& \mathrm{CFID}=6092754 \& \mathrm{CFT}$ OKEN $=63103570$.

Gabelas, J. (2002) Las TIC en la educación. Una perspectiva desmitificadora y práctica sobre los entornos de aprendizaje generados por las nuevas tecnologías. Fecha de última consulta 7 de Diciembre de 2004.

http://www.uoc.edu/web/esp/art/uoc/ gabelas0102/gabelas0102.html

Graves, W. (2001). The new challenges of E-learning. Ubiquity. Consultado en mayo 18, 2005 en http:// doi.acm.org/10.1145/359426.359429.

Hentea, M. \& Shea, M. \& Pennington, L. (2003). A perspective on fulfilling the expectations of distance education. Último acceso mayo 2, 2005. http://doi.acm.org/10.1145/947121.947158.

Masie, E. (2007). El desarrollo de e-learning en Lationoamérica. Último acceso diciembre 7 , 2007. www.antargeo.cl/observatorio/images/ elearning/elliot.pdf

Olivera, A. (s/f) . Desarrollo de proyectos de e-learning. Fecha de última consulta 10 de Noviembre de 2004. Disponible en:

http://www.aldeaeducativa.com/aldea/tareas2. asp? which $=1389$
Rodríguez, J. (2000) Los nuevos aprendizajes virtuales y sus consecuencias. Fecha de última consulta 17 de Octubre de 2004. Disponible en:

http://www.uaambit.org/jornades2000/ Ponencias/j00-illera.htm

Sangrá, A. La calidad en las experiencias virtuales de educación superior. Fecha de última consulta 5 de Diciembre de 2004. Disponible en:

http://www.edulab.net/cast/virtual.pdf

Shoniregun, C. \& Gray, S. (2003). Is E-learning really the future or a risk? Commun. ACM Último acceso mayo 2, 2005.

http://doi.acm.org/10.1145/777947.777948.

Sigalés, C. (2002). El potencial interactivo de los entornos virtuales de enseñanza y aprendizaje en la educación a distancia. Ponencia publicada en el sitio web de la Universidad Abierta de Cataluña. Fecha de última consulta 10 de Diciembre de 2004. Disponible en:

http://www.uoc.edu/web/esp/art/uoc/ sigales0102/sigales0102.html

Smith, T. and L. Zeng, Marcia and Agapova, Olga and Buchel, Olha and Freeston, Michael and Frew, Jim and Hill, Linda and Smart, Laura and Tierney, Tim and Ushakov, Alex (2002). Educational computing: myths versus methods —-why computers haven't helped and what we can do about it.

Último acceso abril 28, 2005. http://doi.acm. org/10.1145/97344.97413.

Weil, Jorge. (s/f). La Universidad Virtual, la enseñanza no presencial y el nuevo paradigma educativo. Ponencia presentada en las III Jornadas de Educación a Distancia - "Mercosur 99". Fecha de última consulta 10 de Diciembre de 2004.

Disponible en: http://www.edudistan.com/ ponencias/Jorge \%20Weil.html

Zhang, D. y Zhao and J. Leon and Zhou, Lina and Nunamaker Jr., Jay F. (2004). Can e-learning replace classroom learning? Commun. ACM. Último acceso mayo 2, 2005. http://doi.acm. org/10.1145/986213.986216. 